\title{
E-cigarette atomiser resistance
}

Addiction Ontology

\section{Source}

Addiction Ontology

Definition: An e-cigarette atomiser quality that is the opposition of its circuit to the flow of current in that circuit.

Informal definition: The electrical resistance of an e-cigarette atomiser which affects how hot it gets when used and therefore how much vapour it produces.

This definition was imported from the Addiction Ontology (see https://addictovocab.org/). Comments and suggestions for improvements are welcome using the Qeios review system.

Definitions imported from the Addiction Ontology (AddictO) are what are known as 'ontological definitions'. See this article in Qeios for an explanation https://www.qeios.com/read/YGIF9B.

Ontological definitions can sometimes be hard to read. In those cases we also include an informal definition.

Definitions also often require elaboration to make it clear how they should be used and what they include. In those cases we include a comment.

Also, definitions sometimes require an explanation as to how they came about to help users understand how they relate to alternative definitions. In those cases we include a curator note. 